\title{
Mamdani Inference Technique for prediction of egg hatching parameters of different species of birds
}

\author{
Wellington Makondo ${ }^{1}$, Kudzanayi Chiteka, Gladman Jekese, Larry Chikukura ${ }^{2}$, Weston Govere ${ }^{3}$ \\ ${ }^{1}$ Department of Information Technology, Department of Industrial and Manufacturing Engineering \\ Harare Institute of Technology, Zimbabwe \\ ${ }^{2}$ Centre for Environmental Sciences and Climate Resilient Agriculture \\ Indian Agricultural Research Institute, New Delhi, India \\ ${ }^{3}$ Department of Basic Sciences, Midlands State University - Manicaland College of Applied Sciences, Mutare Zimbabwe \\ \{wmakondo, kchiteka, gjekese\}@hit.ac.zw, chikslarry@gmail.com, wgovere@msu.ac.zw
}

\begin{abstract}
Identification of the size of hatching eggs is a vital factor in predicting the type of bird as well as temperature and humidity parameters to the success of the incubator. The egg hatching incubator works on the principle that all eggs are of same type of bird and the conditions would be conducive for every egg to hatch. An automatic egg hatching temperature and humidity control method was proposed based on Mamdani inference technique to hatch different species of birds. In order for the temperature and humidity to be predicted using fuzzy logic, some rules should be stemmed from experience of the experts. Such rules are stated through facts about normal body temperatures of different birds. The classification accuracy reached $100 \%$ for all types of birds being small, medium or large. Mamdani inference technique is feasible, robust and has generalization ability leading to accurate prediction of temperature, humidity and hatching period.
\end{abstract}

Keywords: Fuzzy logic, Temperature, Humidity, Incubator, Egg hatching

\section{Introduction}

Incubators are substitute for hens in the poultry industry to hatch chicks. It is a device used to grow and maintain microbiological cultures through maintenance of optimal temperature and humidity. Higher hatch rates are achieved due to the ability to control both temperature and humidity. Temperature, relative humidity and oxygen concentration are key factors in the hatching process to achieve satisfactory hatching rate. Incubation system is a multi-interference, multi-variable and time delay complex self-motivated system. It is hard to control well in conventional control methods [1].

Nevertheless, temperature, relative humidity and oxygen concentration of accurate control is essential to improve quality and hatchability of chicks [2]. Quite a number of studies have been dedicated to the control of incubators, for example in [3], they provided a fuzzy expert decoupling design methods and realized the intelligent control of an incubator. A classical control method was proposed which developed a fuzzy control system for temperature and humidity [4]. Tao and Kaixue [5] described Fuzzy-PID dual-mode control in relation to temperature and humidity for incubation. In the breeding cycle of the commercial birds, the bird normally begins to lay small eggs and in few weeks, will go to medium size and then to the preferred large size egg. Though egg size can be influenced using fat levels, enzymes and protein, some factors such as body weight of the bird and age, yolk weight and nutrient intake can control egg size [6].

Shape examination of hatching eggs is imperative and hard work in farms. Physical inspection lacks objectivity and is timewasting. A method of automatic shape identification was proposed based on moment technique, machine vision and enhanced GA-NN algorithm [7].

Asuquo and Okon [8] studied the effects of egg size on fertility and age in lay and hatchability of eggs. They observed that chicken egg size within the range of 45-56 g would hatch better than small eggs. Senapati et al., [9] reported positive correlation between hatchability and egg weight. A correlation between hatching and egg weight in domestic birds has also been discovered elsewhere [10]. In the context of life-history theory, egg size has been widely studied because it can be highly variable. Some previous studies have shown that egg size can affect both parental and offspring fitness [11].

Machine vision for the production of vaccines introduced hatching egg fertility detection and image processing presented a method to detect the middle-stage hatching egg fertility. Multi-scale morphological transformation was used to improve the image, and then recognize the quality of the shade of the egg. The image was locally segmented so as to extract major embryo blood-vessel features of the embryo image, and then the Weight Fuzzy

ACRID 2017, June 20-21, Victoria Falls, Zimbabwe

Copyright $(\mathcal{2} 2017$

DOI 10.4108/eai.20-6-2017.2270709 
C-means clustering algorithm based on histogram calculated the threshold. The number of the blood-vessels was counted to detect the fertility [12].

Temperature sensor and humidity sensor was selected to compose sensor groups after evaluating hatching condition, the system monitors and controls temperature and humidity automatically through 1 -wire bus technology. The sensor drives actuators to realize temperature, humidity and turning-over-eggs control. Hatching experts recommended $38.2^{\circ} \mathrm{C}$ temperature during starting-hatching, $0 \sim 4$ days, $38.0^{\circ} \mathrm{C}$ in $5 \sim 7$ days, $37.8^{\circ} \mathrm{C}$ in $8 \sim 12$ days, $37.6^{\circ} \mathrm{C}$ in $13 \sim 15$ days, $37.4^{\circ} \mathrm{C}$ in $16 \sim 19$ days, $37.2^{\circ} \mathrm{C}$ in $20 \sim 21$ days [13].

The humidity demand is 53\% relative humidity in 1 3 days during starting hatching, $58 \%$ relative humidity in $4 \sim 6$ days, $55 \%$ relative humidity in $7 \sim 18$ days, $65 \%$ relative humidity on the 19 th day, $70 \%$ relative humidity on the 20 th day, $65 \%$ relative humidity on the 21 st day. If the setting value is more than the humidity, then starts humidifier, which can increase thrifty rate of day-old chicks and sustain clean health [13].

Hatching control system has stringent constraint on temperature and humidity, and only right temperature and humidity can increase hatching rate and rate of chicks, which can attain good economic efficacy.

In [14], they used a single chip microcomputer as the incubator core which makes use of temperature sensor and humidity sensor for examination of temperature and humidity in the incubator. The microcomputer splits the complex incubation controlling system into heating fuzzy and adding wet fuzzy controlling system so that the fuzzy control of temperature and humility can be achieved. The system responds swiftly and has high index of controlling accuracy that results in improved incubation rate.

In this paper, we present an intelligent egg hatching temperature and humidity control method based on Mamdani inference technique to hatch different species of birds. The proposed method covered only three types of birds that are quail, chicken and duck.

\section{Methodology}

In this research, three bird egg species were used that is quail, chicken and duck. The egg weights and sizes were obtained from the related work and classified as shown in Table 1. These were used as the input parameters into the fuzzy system. The egg sizes were classified as small, medium and large and the weights were classified as light, average and heavy. The expected outputs were predetermined as humidity, temperature, period and bird classification shown in table 2 .

Table 1. Egg weights and sizes of different bird species classification

\begin{tabular}{|l|l|l|l|l|}
\hline & \multicolumn{2}{|c|}{ Egg size (cm) } & \multicolumn{2}{c|}{ Egg weight (g) } \\
\hline Quail & $2.5-3.0$ & Small & $9-10.5$ & Light \\
\hline Chicken & $5.0-6.0$ & Medium & $45-56$ & Average \\
\hline Duck & $7.0-9.0$ & Large & $70-110$ & Heavy \\
\hline
\end{tabular}

Table 2. Hatching Parameters

\begin{tabular}{|l|l|l|l|l|l|l|}
\hline & \multicolumn{2}{|c|}{ Value \& Fuzzy Terms } & \multicolumn{2}{c|}{ Value \& Fuzzy Terms } & \multicolumn{2}{c|}{ Value \& Fuzz Terms } \\
\hline Humidity & $40-50$ & Low & $50-60$ & Medium & $85-95$ & High \\
\hline Temperature $\left({ }^{\circ} \mathrm{C}\right)$ & $41.5-42.5$ & Warm & $41-45$ & Warm-Hot & $104-106$ & Hot \\
\hline Period (Days) & $16-17$ & Short & $19-23$ & Average & $25-28$ & Long \\
\hline Classification & $1-2$ & Quail & $2.1-3$ & Chicken & $3.1-4$ & Duck \\
\hline
\end{tabular}

For egg size and weight, the Gaussian membership functions were used.

$$
f(x ; \sigma, c)=e^{\frac{-(x-c)^{2}}{2 \sigma^{2}}}
$$

A Gaussian membership function is determined complete by $\mathrm{c}$ and $\sigma$; $\mathrm{c}$ represents the MFs centre and $\sigma$ determines the MFs width. 
For temperature, humidity and period, trapezoidal membership functions were used.

$$
f(x ; a, b, c, d)=\max \left(\min \left(\frac{x-a}{b-a}, 1, \frac{d-x}{d-c}\right), 0\right)
$$

The parameters $\{\mathrm{a}, \mathrm{b}, \mathrm{c}, \mathrm{d}\}$ (with $\mathrm{a}<\mathrm{b}<=\mathrm{c}<\mathrm{d}$ ) are the $\mathrm{x}$ coordinates of the 4 corners of the trapezoidal membership function.

Lastly, on classification, the triangular membership function was used.

$$
f(x ; a, b, c)=\max \left(\min \left(\frac{x-a}{b-a}, \frac{c-x}{c-b}\right), 0\right)
$$

The parameters $\{\mathrm{a}, \mathrm{b}, \mathrm{c}, \mathrm{d}\}$ (with $\mathrm{a}<\mathrm{b}<\mathrm{c}$ ) are the $\mathrm{x}$ coordinates of the 3 corners of the triangular membership function.

In this system, fuzzy rules used were based on the human knowledge. The following rules were expressed for representing a trained system.

Rule 1: If weight is light and egg size is small then temperature is warm, humidity is medium, period is short and class is Quail.

Rule 2: If Weight is Average and Size is Medium then Temperature is Warm-Hot, Humidity is Low, Period is Average and Class is Chicken.

Rule 3: If Weight is heavy and Size is large then Temperature is Hot, Humidity is High, Period is long and Class is Duck.

The Mamdani inference technique was used for defuzzification.

$$
\text { Crisp Output }=\frac{\sum \mu x}{\sum \mu}
$$

\section{Results}

The simulation model for the hatcher was successfully developed. The simulation results showed that the model could accurately determine the required conditions for hatching different types of eggs. Testing was done using the input data in Table 3 and the corresponding predicted outputs are shown in the table. The results showed that the system could determine the most suitable conditions for a particular type of eggs even if the input weight and size are outside the training values. This is shown by means of egg size of $8.5 \mathrm{~cm}$ together with egg weight of $65 \mathrm{~g}$. The system was intelligent enough to tell that this egg would probably fall under the classification of duck which would be the case if a human being is to give a verdict.

Table 3: Inputs and corresponding predicted outputs

\begin{tabular}{|l|l|l|l|l|l|}
\hline \multicolumn{2}{|c|}{ Inputs } & \multicolumn{5}{c|}{ Outputs } \\
\hline Size $(\mathrm{cm})$ & Weight $(\mathrm{g})$ & Temperature $\left({ }^{\circ} \mathrm{C}\right)$ & Humidity & Period (Days) & Classification \\
\hline 2.8 & 9.94 & 42 & 55.1 & 17 & 1.5 (Quail) \\
\hline 5.75 & 59 & 43.5 & 46.1 & 21.9 & 2.52 (Chicken) \\
\hline 9 & 80 & 105 & 90 & 28 & 3.51 (Duck) \\
\hline 8.5 & 65 & 105 & 90.1 & 28 & 3.51 (Duck) \\
\hline 2.5 & 13 & 42 & 55.1 & 17 & 1.51 (Quail) \\
\hline
\end{tabular}




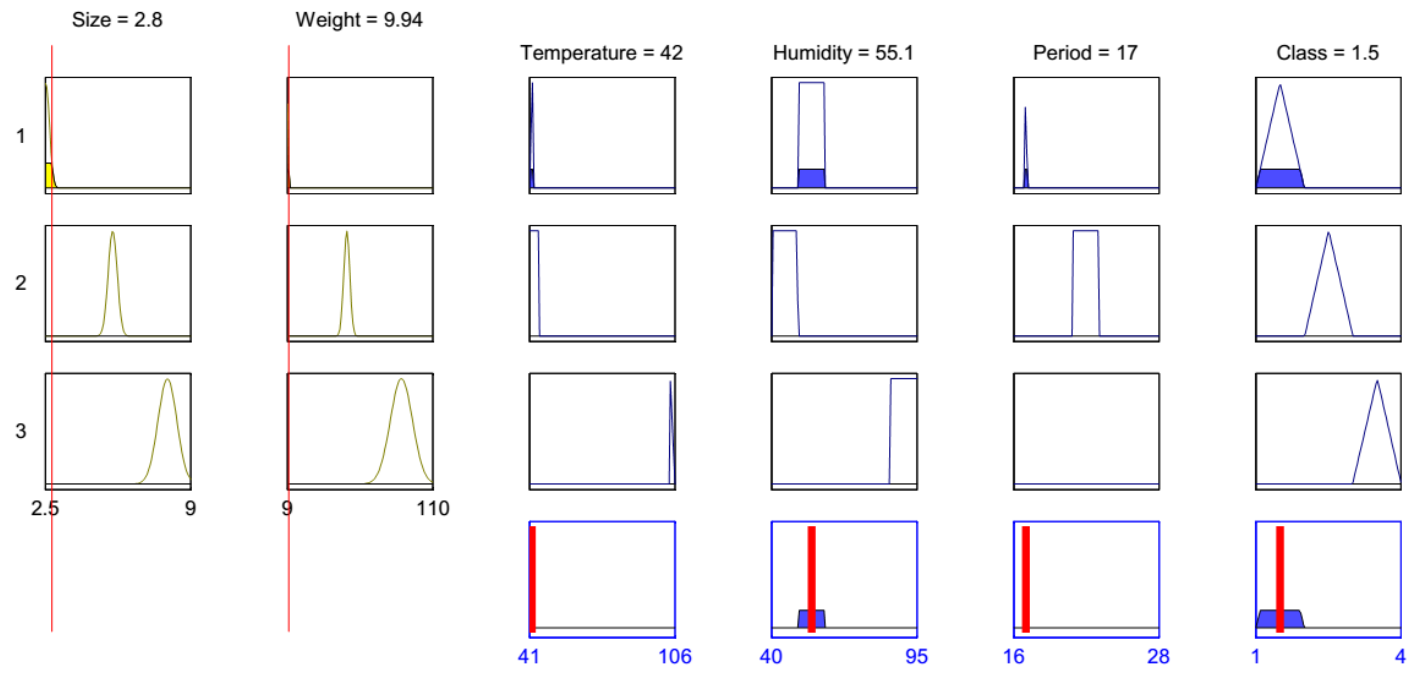

Figure 1. Predicted hatching parameters for a Quail

In figure 1, egg size of $2.8 \mathrm{~cm}$ and weight of $9.94 \mathrm{~g}$ has been classified as an egg of a quail as well as $42{ }^{\circ} \mathrm{C}$ temperature, 55.1 humidity and hatching period of 17 days were predicted by the fuzzy inference system.
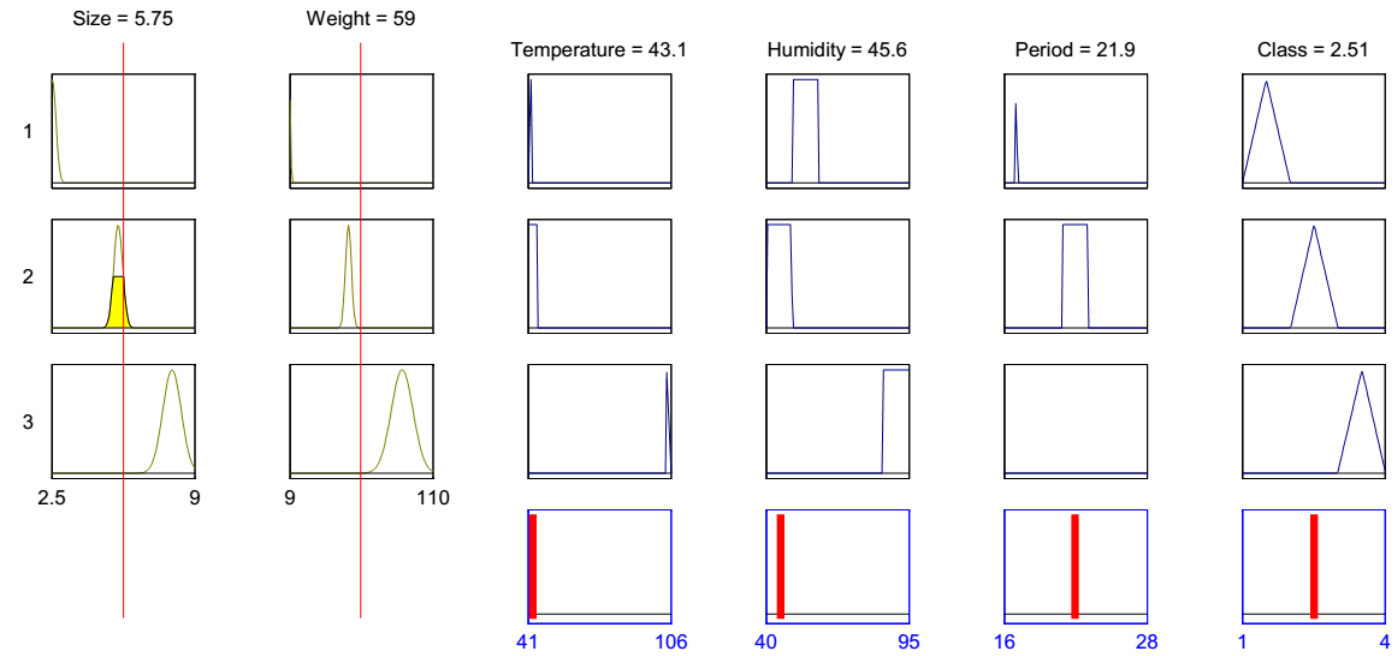

Figure 2. Predicted hatching parameters for a Chicken

The following inputs were used in figure 2, egg size of $5.75 \mathrm{~cm}$ and weight of $59 \mathrm{~g}$ has been categorized as a chicken egg as well as $43.1^{\circ} \mathrm{C}$ temperature, 45.6 humidity and hatching period of 21.9 days were predicted by the fuzzy inference system. 


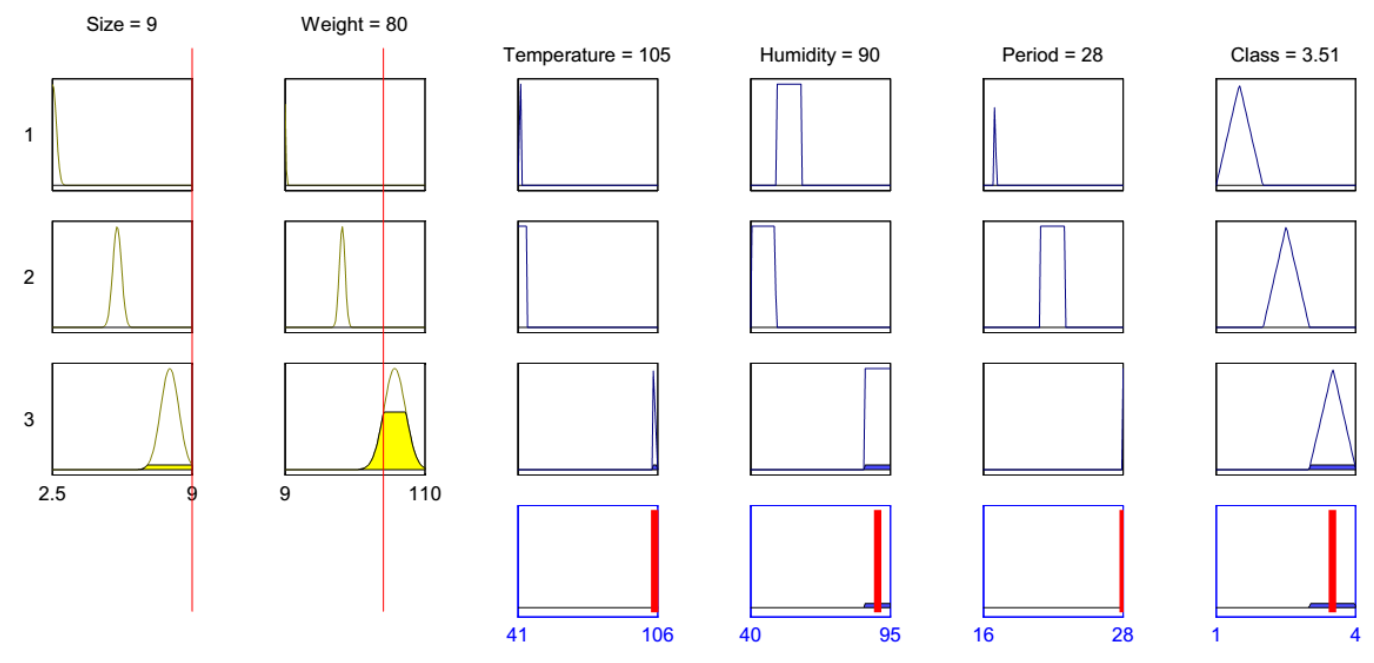

Figure 3. Predicted hatching parameters for a Duck

Egg size of $9 \mathrm{~cm}$ and weight of $80 \mathrm{~g}$ were used as inputs in figure 3. The fuzzy inference system predicted the inputs to be a chicken egg as well as $105^{\circ} \mathrm{C}$ temperature, 90 humidity and hatching period of 28 days.

\section{Conclusion}

The simulated results of incubation parameters for different types of birds using Mamdani inference technique integrates the capabilities of chicken, duck and quail hatchers into a single incubator. The hybrid incubator system is convenient for farmer breeding birds at different intervals. Accurate hatching parameters prediction can only be made when same bird type of eggs are in the incubator otherwise the hatching rate could be adversely affected. In future, a way of alerting the users of the incubator about bird type egg inconsistencies should be implemented.

\section{References}

1. Wu Shu-ci; Zhou Guo-xiong; Yan Mi-ying; Zhou Kai; Zheng Rui-ling, "Research of hybrid intelligent control for incubation," Control, Automation, Robotics and Vision, 2008. ICARCV 2008. 10th International Conference on, vol., no., pp.2026, 2030, 17-20 Dec. 2008.

2. Anna Valros, Reeta Vuorenmaa, Andrew M Janczak. "Effect of simulated long transport on behavioural characteristics in two strains of laying hen chicks". Applied Animal Behaviour Science, 2008，109(1):58 67

3. Wang Wanliang, Wu Qidi, Zhao Yanwei, et al. "Multivariable fuzzy expert controller for poultry incubator" [J]. Transactions of the Chinese Society for Agriculture Machinery, 1998, 29 (3):84 89

4. Cai bing. "Design and Realization of Intelligent Control System for Incubation". Journal of UEST of China, 2004,33 (2): 188 191

5. Su Tao, Yao Kaixue. “The Fuzzy-PI-based dual-mode control system” J. Control Engineering of China, 2005, 7 (12): 145 148

6. Abiola, S.S., O.O Meshiove, B.O Oyerinde and M.A Bamgbose, 2008. Effect of egg size on hatchability of broiler chicks. Arch Zootech., 57:83-86.

7. Zhi-hong Yu; Chun-guang Wang; Jun-qing Feng; Yang Li, "Study on Automatic Shape Identification of Hatching Eggs Based on an Improved GA Neural Network," Natural Computation, 2008. ICNC '08. Fourth International Conference on , vol.2, no., pp.575,578, 18-20 Oct. 2008

8. Asuquo, B.O. and B. Okon. 1993. "Effects of age in lay and egg size on fertility and hatchability of chicken eggs". Nig. J. of Anim. Prod., 20: 122- 124.

9. Senapati, P.K, K.G. Dask Madal and A.K. Chatterjee. 1996. "Relationship between egg weight, shape index, fertility and hatchability of Japanese quail eggs". Environ. Ecol. Stat., 14: 574- 577.

10. Abiola, S.S. 1999. "Effects of turning frequency of hen's egg in electric table-type incubator on weight losses, hatchability and mortality". Nig. Agr. J., 30: 77-82.

11. Williams, T.D. 1994. "Intraspecific variation in egg size and egg composition in birds: effects on offspring fitness". Biol. Rev., 68: 35-39. 
12. Baoming Shan, "Fertility Detection of Middle-stage Hatching Egg in Vaccine Production Using Machine Vision," Education Technology and Computer Science (ETCS), 2010 Second International Workshop on , vol.3, no., pp.95,98, 6-7 March 2010

13. Wen Xinling, "Avian Hatching Monitor and Control System Based on 1-Wire Sensor Groups," Education Technology and Training, 2008 and 2008 International Workshop on Geoscience and Remote Sensing. ETT and GRS 2008. International Workshop on, vol.1, no., pp.357,360, 21-22 Dec. 2008

14. Jiang Huiping, Zhou Guoxiong. "Application of Single Chip Microcomputer in the Incubator's Temperature and Humility Controlling System”, Journal of Hunan University of Science and Engineering, Vol. 28, Apr.2007, pp. 25-26. 